\title{
CARACTERIZACIÓN DE LA ESTRATA HERBÁCEA EN PRADERAS Y BOSQUES DE ÑIRRE (Nothofagus antarctica) EN LA PATAGONIA SUR DE CHILE
}

\author{
Alonso, Máximo²; Balocchi, Oscar ${ }^{3}$ y Schmidt, Andreas ${ }^{4}$
}

\section{RESUMEN}

Los bosques de Nothofagus antarctica de la Patagonia Sur de Chile son muy valorados tanto por su rol ecológico como por su uso ganadero, el cual se remonta a la colonización de la región. A pesar de lo anterior, no existe suficiente información sobre las características productivas de la estrata herbácea asociada a ellos, lo que dificulta su manejo silvopastoril. Así, el objetivo de este trabajo fue estudiar el efecto de distintos niveles de cobertura arbórea sobre las características de la estrata herbácea asociada a los bosques de $N$. antarctica (ñirre) en la región de Magallanes.

Se establecieron 4 ensayos a lo largo de un transecto de $100 \mathrm{~km}$ en la Patagonia Sur Chilena. En cada ensayo se identificaron sectores (condiciones) de pradera y bosques con diferentes niveles de cobertura. Las precipitaciones en los ensayos fluctúan entre los 398 y $486 \mathrm{~mm}$ al año. La temperatura mínima de $0{ }^{\circ} \mathrm{C}$ se produce en Julio y la máxima de $11{ }^{\circ} \mathrm{C}$ en Enero. Los suelos corresponden a materiales fluvio-glacio-volcánicos desarrollados en un relieve plano a ligeramente inclinado de 30 a $60 \mathrm{~cm}$ de profundidad. Los bosques de $N$. antarctica se encuentran en distintas fases de desarrollo, con alturas dominantes entre 5 y $14 \mathrm{~m}$. En cada ensayo y condición se establecieron en forma aleatoria múltiples cuadrantes de $1 \mathrm{~m}^{2}$. En los sectores sometidos a pastoreo en forma regular se establecieron jaulas metálicas de $1 \mathrm{~m}^{2}$ a manera de exclusiones. Se determinó la composición botánica y el rendimiento de materia seca (MS) en cada cuadrante y jaula de exclusión.

Los resultados obtenidos indican un rendimiento de la estrata herbácea equivalente a $1.834,1.842$ y $1.131 \mathrm{~kg}$ MS por hectárea para praderas, bosques abiertos y semidensos, respectivamente, no habiendo diferencias significativas en la producción de MS entre praderas y bosques abiertos. Sin embargo, la condición de bosque abierto presentó el mayor porcentaje de especies de alto valor forrajero, como Trifolium repens, Dactylis glomerata y Holcus lanatus. La pradera en cambio, presentó mayor porcentaje de Agrostis capillaris y de malezas de importancia agronómica. Se concluye que bosques de $N$. antarctica con coberturas arbóreas $<50 \%$ presentan un gran potencial de uso silvopastoril, dado por el rendimiento y calidad de la pradera asociada a ellos.

Palabras clave: Composición botánica, Estrata herbácea, Patagonia

\section{SUMMARY}

Nothofagus antarctica forests in the Southern Chilean Patagonia are valuable resources by their ecological role and the cattle grazing use, which goes back to the colonization of the region. However, there is not enough information about the productive characteristics of the understory associated to them, which makes difficult their silvopastoral management. Thus, the objective of this work was to study the effect of different tree cover levels on the characteristics of the understory associated to $N$. antarctica (Nirre) forests in the Magallanes Region.

\footnotetext{
2 Ingeniero Agrónomo, Ph. D. Instituto de Producción Animal, Facultad de Ciencias Agrarias, Universidad Austral de Chile. Valdivia, Chile. maximo.alonso@uach.cl

3 Ingeniero Agrónomo, Ph. D. Instituto de Producción Animal, Facultad de Ciencias Agrarias, Universidad Austral de Chile. Valdivia, Chile

${ }_{4}$ Ingeniero Forestal, Dr. BIO ${ }^{+}$Consultores. Valdivia, Chile
} 
Four trials were established along a $100 \mathrm{~km}$ transect in the Southern Chilean Patagonia. At each trial open grasslands and forests with different tree cover levels sectors (conditions) were identified. Precipitation at the trials fluctuated between 398 and $486 \mathrm{~mm}$ per year. The minimum temperature of $0{ }^{\circ} \mathrm{C}$ was obtained on July and the maximum temperature of $11^{\circ} \mathrm{C}$ was registered on January. Soils corresponded to fluvio-glacio-volcanic materials developed in a plain to lightly inclined profile of 30 to $60 \mathrm{~cm}$ depth. $N$. antarctica forests were in different stages of development, with dominant tree heights from 5 to $14 \mathrm{~m}$. At each trial and condition multiple $1 \mathrm{~m}^{2}$ quadrats were randomly established. In regularly grazed sectors $1 \mathrm{~m}^{2}$ metalic cages were established. Botanical composition and dry matter (DM) yield at each quadrat and cage were determined.

Results showed an understory yield equivalent to $1.834,1.842$ y $1.131 \mathrm{~kg} \mathrm{DM}$ by hectare in grasslands, open forests and semidense forests of $N$. antarctica, respectively. Differences in DM yield between grasslands and open forests were not significant. However, the understory in open forests showed a higher percentage of high grazing value species like Trifolium repens, Dactylis glomerata and Holcus lanatus. Instead, open grasslands presented a higher percentage of Agrostis capillaris and weeds of agronomic relevance. It is concluded that $N$. antarctica forests with tree covers $<50 \%$ have a high potential for silvopastoral use, based on yield and quality of the understory associated to them.

Keywords: Botanical composition, Patagonia, Understory

\section{INTRODUCCIÓN}

Los bosques de Nothofagus antarctica (G. Forster) Oerst. de la Patagonia Sur de Chile son muy valorados, tanto por su rol ecológico como por su uso ganadero, el cual se remonta a la colonización de la región. Estos bosques corresponden a ecosistemas xerofíticos con precipitaciones anuales entre 300 y $500 \mathrm{~mm}$. Se desarrollan en una amplia gama de condiciones edafoclimáticas, desde suelos con problemas de drenaje a bajas y altas altitudes, sitios expuestos con sustratos inestables, depresiones frías, suelos secos y delgados, hasta las zonas de contacto con la estepa patagónica (Veblen et al., 1996).

En la región de Magallanes los bosques de $N$. antárctica (ñirre) comprenden una superficie superior a las 220.000 ha y gran parte de ellos se encuentran en zonas de contacto con praderas, en terrenos ubicados a menos de $400 \mathrm{~m}$ de altitud y con pendientes menores al $30 \%$, lo que los hace accesibles para pastoreo gran parte del año (MINAGRI, 2012; SAG, 2003; 2004a y 2004b).

Experiencias de manejo silvopastoril a nivel internacional (Bahamonde et al., 2012; Barnes et al., 2011; Benavides et al., 2009; Peri, 2005; Peri, 2008; Peri, 2009) han demostrado que la presencia de una estrata arbórea produce un fuerte aumento en la disponibilidad de forraje, prolonga la curva de crecimiento de la pradera asociada a esta e influye favorablemente en el comportamiento animal, no solo por la mayor disponibilidad de alimento, sino también por las mejores condiciones ambientales que le permiten a los animales ahorrar energía, que de otro modo utilizarían en termorregulación, para destinarla a producción.

Ensayos de manejo silvopastoril realizados en bosques de ñirre de las provincias de Magallanes y de Última Esperanza (Schmidt, 2008; Schmidt et al., 2013; Schmidt y Alonso, 2016), confirman el valor de estos bosques y las ventajas de su manejo con criterios silvopastoriles.

A pesar de ello, no existe suficiente información sobre las características productivas de la estrata herbácea asociada a estos bosques, lo que dificulta su manejo silvopastoril y pone en riesgo su regeneración. Así, el objetivo de este trabajo fue estudiar el efecto de distintos niveles de cobertura arbórea sobre las características de la estrata herbácea asociada a bosques de $N$. antarctica (ñirre) en la Región de Magallanes. 


\section{MATERIAL Y MÉTODO}

Se establecieron 4 ensayos a lo largo de un transecto de aproximadamente $100 \mathrm{~km}$ de longitud en la Patagonia Sur Chilena (Figura Nº 1).

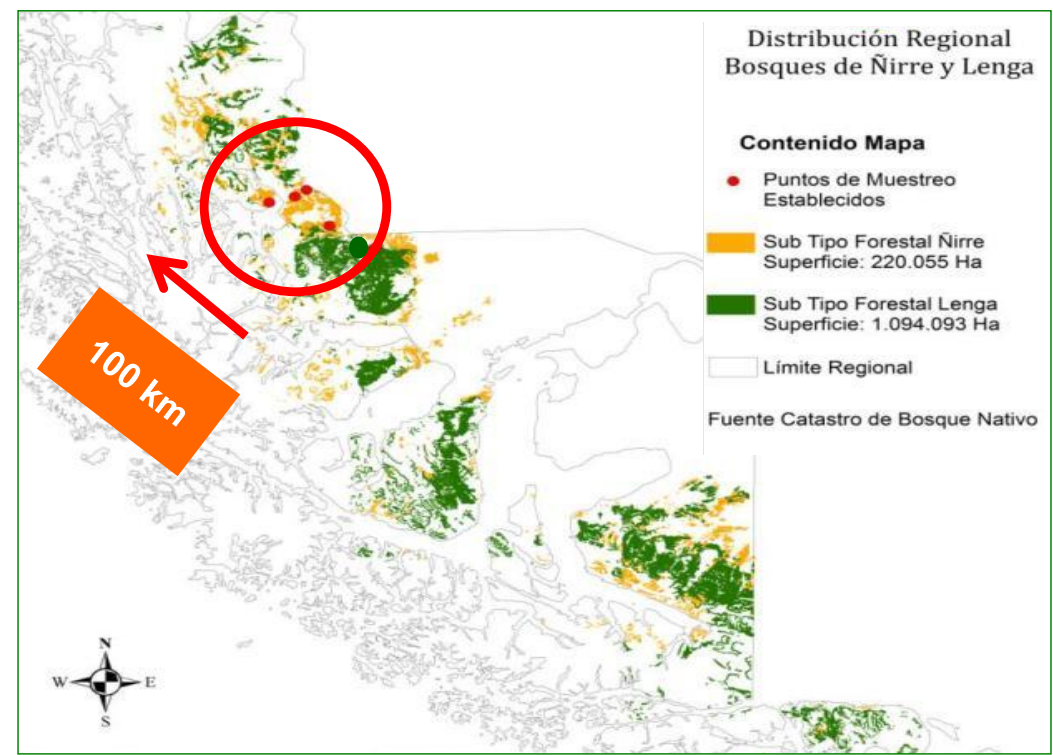

Figura $\mathbf{N}^{\circ} 1$

UBICACIÓN DE LOS ENSAYOS DE SILVOPASTOREO EN MAGALLANES

Los bosques de $N$. antarctica se encuentran en distintas fases de desarrollo, con alturas dominantes entre los 5 y $14 \mathrm{~m}$ (Figura $\mathrm{N}^{\circ} 2$ ). La temperatura mínima de $0{ }^{\circ} \mathrm{C}$ se produce en julio y la máxima de $11^{\circ} \mathrm{C}$ en enero.

Tranquilo: Corresponde a un bosque puro de ñirre históricamente sometido a pastoreo por bovinos, con una altura de árboles dominantes de $13 \mathrm{~m}$. De acuerdo a la información climática disponible en la base de datos de WorldClim (www.worldclim.org), las precipitaciones en el sector donde se ubica el ensayo bordean los $431 \mathrm{~mm}$ anuales.

El Palenque: Corresponde a un sector con bosques puros de ñirre, con una altura de árboles dominantes de $8 \mathrm{~m}$. Gran parte de los bosques en esta área fueron quemados durante la primera mitad del siglo XX para habilitar praderas para la ganadería. Actualmente, el sitio del ensayo es sometido a pastoreo por ganado bovino y guanacos. De acuerdo a la información climática disponible en WorldClim (www.worldclim.org), las precipitaciones en el sector bajo estudio alcanzan los $416 \mathrm{~mm}$ anuales.

Los Cerritos: Corresponde a un sector con bosques puros de ñirre, con una altura de los árboles dominantes de $5 \mathrm{~m}$. En el pasado, este predio ha sido sometido a pastoreo ovino y bovino. En la actualidad se pastorea con ganado bovino. En el predio se han realizado distintos manejos históricos, por lo que es posible encontrar renovales en estado de latizal, sectores de bosque abierto con coberturas arbóreas menores a $30 \%$, praderas arboladas y sectores con fajas. De acuerdo a la información climática disponible en WorldClim (www.worldclim.org), las precipitaciones en el sector donde se ubica el ensayo alcanzan los $398 \mathrm{~mm}$ anuales. 
Península Antonio Varas: Corresponde a un renoval denso de ñirre, con una altura de los árboles dominantes de $14 \mathrm{~m}$ y una abundante estrata arbustiva que se originó a partir de incendios en la primera mitad del siglo XX. En el año 2005 se establecieron cuatro tratamientos de 1 ha: Un testigo, una tala rasa, fajas alternas de ancho variable y un raleo en el que se eliminó el $40 \%$ del área basal, bajando de 1.830 a 560 árboles por hectárea y dejando un $40 \%$ de cobertura arbórea. Desde su establecimiento el año 2005, la superficie de este ensayo no ha sido sometida a pastoreo. De acuerdo a la información climática disponible en WorldClim (www.worldclim.org), las precipitaciones en el sector del ensayo bordean los $486 \mathrm{~mm}$ anuales.

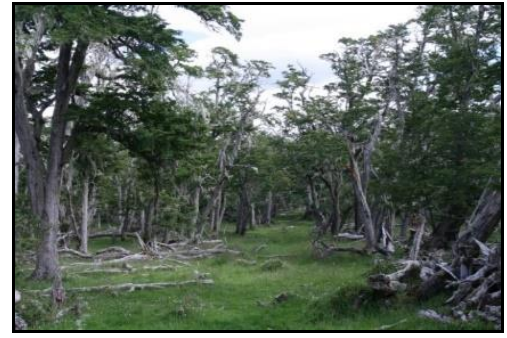

Tranquilo

$13 \mathrm{~m}, 431 \mathrm{~mm} / \mathrm{año}$

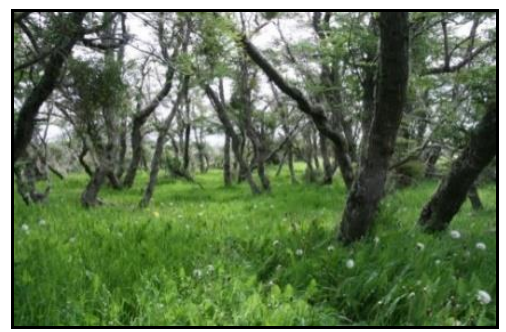

Los Cerritos

$5 \mathrm{~m}, 398 \mathrm{~mm} / \mathrm{año}$

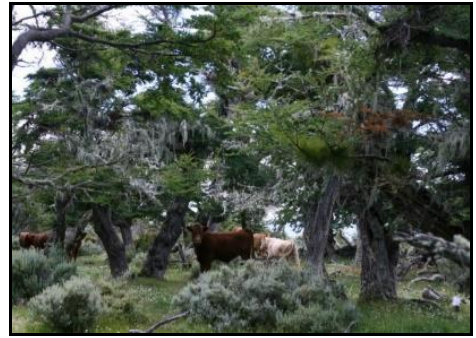

El Palenque

$8 \mathrm{~m}, 416 \mathrm{~mm} / \mathrm{año}$

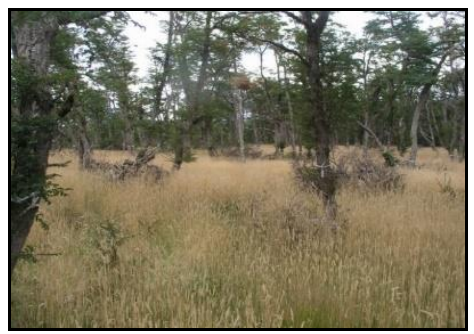

Península Antonio Varas

$14 \mathrm{~m}, 486 \mathrm{~mm} / \mathrm{año}$

Figura $\mathrm{N}^{\circ} 2$

ENSAYOS DE SILVOPASTOREO EN MAGALLANES

Los suelos de los sitios de muestreo se desarrollan en un relieve plano a ligeramente inclinado y han evolucionado a partir de materiales fluvio-glacio-volcánicos. En general, estos suelos se caracterizan por presentar un drenaje imperfecto provocado por un abrupto cambio textural o bien por la presencia de capas compactadas entre los 40 y $60 \mathrm{~cm}$ de profundidad. El subsuelo (Horizontes C), generalmente compacto, está constituido por till glaciar. Figuras redoximórficas características de drenajes impedidos son frecuentes de observar en los horizontes sub-superficiales $\mathrm{C}$. Esto último, es más notorio en las localidades de mayor precipitación (Schmidt, 2008).

Los perfiles de suelos en los sitios de muestreo son similares en cuanto a los horizontes y sus espesores promedio. En los distintos sitios de muestreo los horizontes A y Ap son poco profundos, variando entre los 5 y $8 \mathrm{~cm}$ de espesor. Los horizontes sub-superficiales $A B$ o $B$ son poco evolucionados (Bw), presentan espesores entre 20 y $35 \mathrm{~cm}$, y generalmente constituyen el límite para el desarrollo radicular de la vegetación arbórea. Las texturas en general varían desde franco a franco-arenosas, aumentando en el caso de Antonio Varas el contenido de arcillas en profundidad (Horizonte C). Este tipo de texturas hace inferir que los suelos presentan una buena permeabilidad (Schmidt, 2008). 
Los suelos son fuertemente a moderadamente ácidos presentando valores de $\mathrm{pH}\left(\mathrm{H}_{2} \mathrm{O}\right.$ $1: 1)$ entre 4,6 y 6,1 . El contenido de materia orgánica del suelo sigue una tendencia normal con una marcada disminución en profundidad en todas las situaciones y coberturas analizadas. Diferencias entre los sitios de estudio son perceptibles al comparar condiciones más áridas, como Palenque y Cerritos, con las de mayor precipitación efectiva, como Tranquilo y Antonio Varas (Schmidt, 2008).

Los sectores de Antonio Varas y Tranquilo presentan los mayores contenidos de NPK aprovechables, preferentemente en los primeros $10 \mathrm{~cm}$ de suelo y con tendencias a una disminución en profundidad. Las coberturas medias y bajas tienden a presentar mayores concentraciones de NPK aprovechables. EI N aprovechable sigue una tendencia en función de los contenidos de materia orgánica. El $\mathrm{N}$ aprovechable en los ensayos corresponde a un nivel medio (25-50 ppm). En términos de $\mathrm{P}$ aprovechable, este tiende a presentarse mayoritariamente en niveles bajos (7-15 ppm) a muy bajos $(<7 \mathrm{ppm})$. Solo en los primeros $10 \mathrm{~cm}$ del suelo en Tranquilo, el $\mathrm{P}$ aprovechable está en cantidades adecuadas para el óptimo crecimiento de la vegetación. El K aprovechable no es un elemento limitante, encontrándose en general niveles altos (>50 ppm) de este elemento (Schmidt, 2008).

En cada ensayo se identificaron sectores (condiciones) de pradera y bosques con diferentes niveles de cobertura. Cada sector de muestreo se caracterizó en términos de la cobertura de copas y la radiación global disponible bajo el dosel arbóreo (Figura $\mathrm{N}^{\circ} 3$ ). La cobertura arbórea y la radiación disponible bajo el dosel arbóreo se determinaron mediante el uso de fotografías hemisféricas.

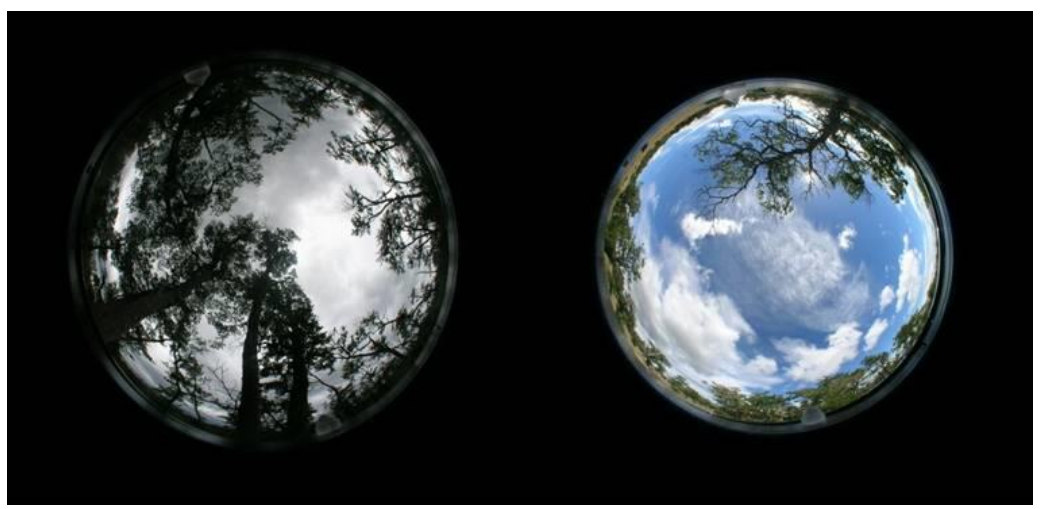

Figura $\mathrm{N}^{\circ} 3$

FOTOGRAFÍAS HEMISFÉRICAS EN RALEO DE ANTONIO VARAS (IZQUIERDA) Y FAJAS EN LOS CERRITOS (DERECHA)

Las fotografías se tomaron en forma sistemática a intervalos de $30 \mathrm{~m}$ en transectos paralelos separados $20 \mathrm{~m}$ unos de otros. Las fotografías se tomaron a 1,2 $\mathrm{m}$ de altura, en condiciones de cielo cubierto siguiendo los protocolos del fabricante del equipo CoolPix 5400, Nikon®, Japan (Delta-T Devices Ltd., United Kingdom). Posteriormente, las fotografías fueron procesadas mediante el software Hemiview® 2.1 (Delta-T Devices Ltd., United Kingdom).

La cobertura arbórea corresponde a la superficie de dosel proyectada verticalmente por unidad de superficie del suelo. El cielo visible corresponde a la proporción de cielo visible en un determinado sector del cielo relativo a su contribución al hemisferio celeste completo. La radiación global corresponde a la suma de la fracción de radiación difusa e indirecta que llega a cada sitio respecto a la que llegaría a un sitio equivalente a cielo abierto, teniendo en cuenta la latitud del sitio (Valladares y Guzmán, 2006). Los niveles de cobertura arbórea para praderas, bosques abiertos y 
semidensos fueron de 0-25, 26-50 y 51-75\%, respectivamente.

En cada ensayo y condición se establecieron en forma aleatoria múltiples cuadrantes de $1 \mathrm{~m}^{2}$. En los sectores sometidos a pastoreo en forma regular se establecieron jaulas metálicas de 1 $\mathrm{m}^{2}$ a manera de exclusiones. Se determinó el porcentaje de suelo desnudo, mantillo o material en descomposición, riqueza de especies, composición botánica y rendimiento de materia seca (MS) de cada cuadrante y jaula de exclusión.

Los resultados fueron sometidos a un ANDEVA y la prueba de comparación de medias de Tukey utilizando JMP® versión 11.

\section{RESULTADOS Y DISCUSIÓN}

El porcentaje de suelo desnudo, mantillo o material en descomposición y cobertura de las distintas especies herbáceas asociadas a los bosques se muestra en el Cuadro $\mathrm{N}^{\circ} 1$.

\section{Cuadro $\mathrm{N}^{\circ} 1$}

PORCENTAJE DE SUELO DESNUDO, MANTILLO Y COBERTURA HERBÁCEA EN PRADERAS Y BOSQUES ABIERTOS Y SEMIDENSOS DE $N$. antarctica EN MAGALLANES

\begin{tabular}{|l|c|c|c|c|c|c|}
\hline \multirow{2}{*}{ Tipo } & \multicolumn{2}{|c|}{ Bosque Semidenso } & \multicolumn{2}{c|}{ Bosque Abierto } & \multicolumn{2}{c|}{ Pradera } \\
\cline { 2 - 7 } & $(\%)$ & EE & $(\%)$ & EE & $(\%)$ & EE \\
\hline Suelo desnudo & $1,56 \mathrm{a}$ & 0,8 & $0,00 \mathrm{~b}$ & 0,0 & $0,00 \mathrm{~b}$ & 0,0 \\
\hline Mantillo & $10,63 \mathrm{a}$ & 1,3 & $13,92 \mathrm{a}$ & 3,8 & $6,76 \mathrm{a}$ & 1,0 \\
\hline Cobertura herbácea* & $87,81 \mathrm{a}$ & - & $86,08 \mathrm{a}$ & - & $93,24 \mathrm{a}$ & - \\
\hline
\end{tabular}

* $100 \%$ - (\% suelo desnudo + \% mantillo). EE: Error Estándar.

Diferentes letras indican diferencias significativas en la prueba de Tukey $(\mathrm{P}<0.05)$

De las tres condiciones de cobertura arbórea estudiadas, la pradera resultó ser aquella con mayor riqueza, con un total de 30 de especies herbáceas. En la estrata herbácea del bosque abierto y del bosque semidenso se encontraron un total de 24 y 23 especies, respectivamente (Cuadro $\mathrm{N}^{\circ} 2$ ).

Respecto de la composición botánica de la estrata herbácea, la condición de bosque abierto presentó un mayor porcentaje de especies de alto valor forrajero como Trifolium repens, Dactylis glomerata y Holcus lanatus, comparada con la pradera y el bosque semidenso.

Por el contrario, la pradera presentó mayor porcentaje de Agrostis capillaris, una gramínea de bajo valor forrajero, y de malezas de importancia agronómica como Hieracium plilosella y Rumex acetosella.

Por su parte, el bosque semidenso presenta una elevada cobertura de Trifolium repens, Blechnum penna-marina y Festuca rubra.

En términos de productividad, los resultados obtenidos indican un rendimiento de la estrata herbácea equivalente a $1.834,1.842$ y $1.131 \mathrm{~kg} / \mathrm{ha}$ de MS para praderas, bosques abiertos y semidensos de $N$. antarctica respectivamente, no habiendo diferencias significativas en la producción de MS entre praderas y bosques abiertos (Figura $N^{\circ} 4$ ). 
Cuadro $\mathrm{N}^{\circ} 2$

PORCENTAJE DE COBERTURA DE ESPECIES HERBÁCEAS PRESENTES EN PRADERAS Y BOSQUES ABIERTOS Y SEMIDENSOS DE $N$. antarctica EN MAGALLANES

\begin{tabular}{|c|c|c|c|c|c|c|}
\hline \multirow{2}{*}{ Tipo } & \multicolumn{2}{|c|}{ Bosque Semidenso } & \multicolumn{2}{|c|}{ Bosque Abierto } & \multicolumn{2}{|c|}{ Pradera } \\
\hline & (\%) & EE & (\%) & EE & (\%) & EE \\
\hline Acaena magellanica & 2,31 & 0,74 & 2,85 & 1,17 & 1,67 & 0,72 \\
\hline Acaena pinnatifida & 1,25 & 1,25 & 0,62 & 0,54 & 0,24 & 0,24 \\
\hline Achillea millefolium & 0,31 & 0,31 & 0,38 & 0,38 & 2,43 & 0,84 \\
\hline Adenocaulon chilense & 1,25 & 0,72 & 1,46 & 0,98 & 0,10 & 0,10 \\
\hline Agrostis capillaris & 2,81 & 2,50 & 8,12 & 2,86 & 28,48 & 4,50 \\
\hline Agrostis magellanica & 0,00 & 0,00 & 0,00 & 0,00 & 4,57 & 2,70 \\
\hline Anemone decapetala & 0,00 & 0,00 & 0,00 & 0,00 & 0,00 & 0,00 \\
\hline Baccharis magellanica & 3,44 & 2,36 & 0,00 & 0,00 & 2,00 & 1,28 \\
\hline Berberis buxifolia & 0,75 & 0,63 & 0,65 & 0,43 & 0,29 & 0,16 \\
\hline Berberis microphylla & 0,13 & 0,13 & 0,00 & 0,00 & 0,00 & 0,00 \\
\hline Blechnum penna marina & 15,31 & 4,09 & 11,35 & 3,75 & 0,14 & 0,10 \\
\hline Bromus coloratus & 0,00 & 0,00 & 0,19 & 0,19 & 0,95 & 0,95 \\
\hline Carex gayana & 0,94 & 0,68 & 0,00 & 0,00 & 0,00 & 0,00 \\
\hline Carex macloviana & 0,00 & 0,00 & 0,00 & 0,00 & 0,10 & 0,10 \\
\hline Cerastium arvense & 0,00 & 0,00 & 0,00 & 0,00 & 1,62 & 0,60 \\
\hline Dactylis glomerata & 0,00 & 0,00 & 2,12 & 1,47 & 0,71 & 0,52 \\
\hline Empetrum rubrum & 0,00 & 0,00 & 0,00 & 0,00 & 0,24 & 0,24 \\
\hline Escallonia rubra & 0,00 & 0,00 & 0,00 & 0,00 & 0,71 & 0,71 \\
\hline Festuca magellanica & 2,06 & 2,06 & 3,19 & 2,15 & 3,10 & 1,84 \\
\hline Festuca rubra & 11,88 & 4,30 & 4,62 & 1,71 & 6,67 & 2,54 \\
\hline Gaultheria mucronata & 5,63 & 4,08 & 7,35 & 2,80 & 0,48 & 0,48 \\
\hline Gentianella magellanica & 0,00 & 0,00 & 0,12 & 0,12 & 0,24 & 0,24 \\
\hline Gunnera magellanica & 0,31 & 0,31 & 3,00 & 2,04 & 0,00 & 0,00 \\
\hline Hieracium patagonicum & 0,31 & 0,31 & 3,54 & 1,40 & 8,71 & 1,88 \\
\hline Hieracium pilosella & 6,56 & 3,78 & 0,19 & 0,19 & 7,86 & 3,57 \\
\hline Holcus lanatus & 2,50 & 1,65 & 6,92 & 2,84 & 2,62 & 1,88 \\
\hline Hypochaeris radicata & 0,00 & 0,00 & 0,69 & 0,58 & 0,00 & 0,00 \\
\hline Luzula alopecurus & 0,00 & 0,00 & 0,00 & 0,00 & 0,29 & 0,24 \\
\hline Musgo spp. & 0,00 & 0,00 & 0,19 & 0,19 & 0,00 & 0,00 \\
\hline Nothofagus antarctica & 0,94 & 0,94 & 0,15 & 0,11 & 0,00 & 0,00 \\
\hline Osmorhiza chilensis & 5,69 & 3,75 & 0,46 & 0,39 & 0,00 & 0,00 \\
\hline Phleum commutatum & 0,06 & 0,06 & 0,00 & 0,00 & 1,05 & 0,74 \\
\hline Poa pratensis & 0,00 & 0,00 & 3,62 & 1,70 & 2,52 & 1,37 \\
\hline Rumex acetosella & 0,50 & 0,50 & 0,00 & 0,00 & 0,90 & 0,40 \\
\hline Senecio patagonicus & 0,00 & 0,00 & 0,00 & 0,00 & 0,95 & 0,56 \\
\hline Taraxacum officinale & 5,31 & 1,67 & 4,08 & 1,63 & 2,24 & 0,81 \\
\hline Trifolium repens & 17,56 & 4,72 & 20,23 & 5,05 & 9,81 & 2,79 \\
\hline Trifolium spadiceum & 0,00 & 0,00 & 0,00 & 0,00 & 1,57 & 0,70 \\
\hline
\end{tabular}

EE: Error Estándar 


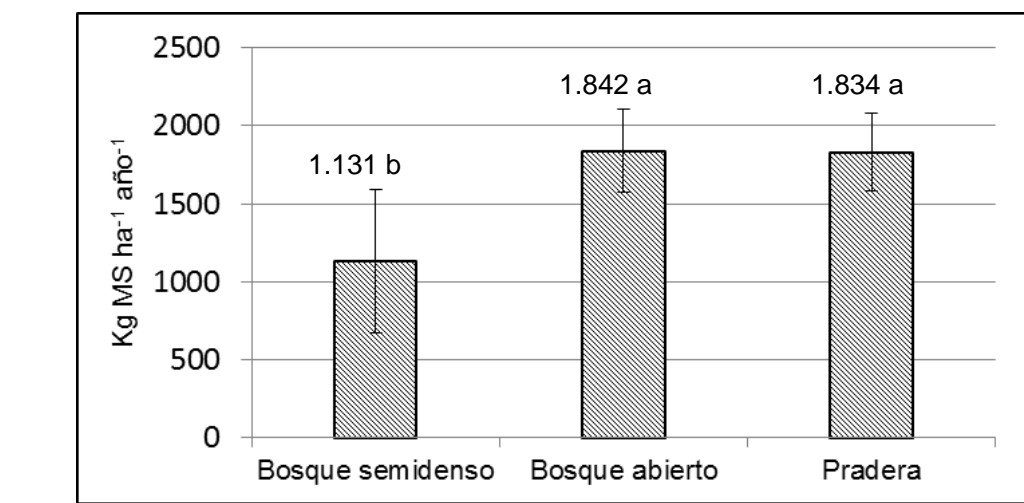

Diferentes letras indican diferencias significativas en la prueba de Tukey $(\mathrm{P}<0.05)$

\section{Figura $\mathrm{N}^{\circ} 4$ \\ RENDIMIENTO DE MS DE LA ESTRATA HERBÁCEA EN PRADERAS, BOSQUES ABIERTOS Y SEMIDENSOS DE $N$. antarctica EN MAGALLANES}

Los resultados obtenidos en este estudio coinciden con aquellos de trabajos realizados en la Patagonia Argentina (Bahamonde et al., 2012; Barnes et al., 2011; Benavides et al., 2009; Peri, 2005; Peri, 2008; Peri, 2009), los cuales han demostrado que la presencia de una estrata arbórea favorece a la estrata herbácea asociada a ella, confirmando la ventaja del manejo de estos bosques con criterios silvopastoriles.

\section{CONCLUSIONES}

Doseles arbóreos con coberturas $<50 \%$ permiten el desarrollo de una estrata herbácea con rendimientos similares a los obtenidos en praderas más expuestas, bordeando las 2 t/ha/año de MS. Sin embargo, la composición botánica de la estrata herbácea en bosques y praderas difiere, aumentando el porcentaje de especies de mayor valor forrajero, como Trifolium repens, Dactylis glomerata y Holcus lanatus, bajo la protección del dosel arbóreo.

Considerando el rendimiento y composición botánica de la estrata herbácea, los bosques de $N$. antarctica con coberturas arbóreas $<50 \%$ (bosques abiertos), presentan un gran potencial para el establecimiento de sistemas silvopastoriles en la región de Magallanes.

\section{RECONOCIMIENTOS}

El presente trabajo es parte del proyecto 029/2013 del Fondo de Investigación del Bosque Nativo (FIBN) de CONAF.

\section{REFERENCIAS}

Bahamonde, H.; Peri, L.; Álvarez, A. y Barnei, A., 2012. Producción y calidad de gramíneas en un gradiente de calidades de sitio y coberturas en bosques de Nothofagus antarctica (G. Forster) Oerst. en Patagonia. Ecología Austral 22:62-73.

Barnes, P.; Wilson, B. R.; Trotter, M. G.; Lamb, D. W.; Reid, N.; Koen, T. and Bayerlein, L., 2011. The patterns of grazed pasture associated with scattered trees across an Australian temperate landscape: An investigation of pasture quantity and quality. Rangeland Journal 33, 121-130. 
Benavides, R.; Douglas, G. and Osoro, K., 2009. Silvopastoralism in New Zealand: Review of effects of evergreen and deciduous trees on pasture dynamics. Agroforestry Systems 76, 327-350

CONAF, 2008. Catastro y Evaluación de Recursos Vegetacionales de Chile.

MINAGRI, 2012. Estrategia para la competitividad del sector agroalimentario y forestal. Región de Magallanes y la Antártica Chilena 2012 - 2020.

Peri, P., 2005. Sistemas Silvopastoriles en Nirantales. IDIA XXI Forestal.

Peri, P., 2008. Respuesta de ovinos a pastizales creciendo en diferentes cobertura de copas en sistemas silvopastoriles de ñire (Nothofagus antarctica) en Patagonia Sur, Argentina. Zootecnia Tropical 26, 363-366.

Peri, P., 2009. Evaluación de pastizales en bosques de Nothofagus antarctica-Método Nirantal Sur, 1er Congreso Nacional de Sistemas Silvopastoriles. INTA, Posadas, Misiones, Argentina, pp. 335-342.

SAG, 2003. El pastizal de Tierra del Fuego. Guía de uso, condición actual y propuesta de seguimiento para determinación de tendencia. Punta Arenas, Chile. 120 p.

SAG. 2004a. El pastizal de Magallanes. Guía de uso, condición actual y propuesta de seguimiento para determinación de tendencia. Punta Arenas, Chile. $132 \mathrm{p}$.

SAG, 2004b. El pastizal de Última Esperanza y Navarino. Guía de uso, condición actual y propuesta de seguimiento para determinación de tendencia. Punta Arenas, Chile. 132 p.

Schmidt, H., 2008. Mejoramiento de la productividad ganadera a través del manejo silvopastoril de los bosques de ñirre en la XII Región. Informe Final Proyecto FIA Código FIA-PI-C-2004-1-F-054. Santiago, Chile. 54 p.

Schmidt. H.; Schmidt, A. y Alonso, M., 2013. Investigación, Mejoramiento, Productividad y Silvopastoreo en la XII Región. Informe Final, Programa FONDEMA Código BIP 30077120-0. Santiago, Chile. 211 p.

Schmidt, A. y Alonso, M., 2016. Manejo silvopastoril de los bosques de ñirre en la XII Región de Magallanes. Informe Final, Proyecto Fondo de Investigación en Bosque Nativo CONAF Código 029/2013. Santiago, Chile. 70 p.

Valladares, F. and Guzmán, B., 2006. Canopy structure and spatial heterogeneity of understory light in an abandoned Holm Oak woodland. Annals of Forest Science 63: 749-761.

Veblen, T. T.; Hill, R. S. and Read, J., 1996. The Ecology and Biogeography of Nothofagus Forests. Yale University Press. New Haven and London. 403 p. 
